Western University

Scholarship@Western

$9-1-2019$

\title{
Eliminating the effects of motion during radiofrequency lesion delivery using a novel contact-force controller.
}

Daniel Gelman

Allan C Skanes

Douglas L Jones

Michael Timofeyev

Tal Bar-On

See next page for additional authors

Follow this and additional works at: https://ir.lib.uwo.ca/biophysicspub

Part of the Medical Biophysics Commons

\section{Citation of this paper:}

Gelman, Daniel; Skanes, Allan C; Jones, Douglas L; Timofeyev, Michael; Bar-On, Tal; and Drangova, Maria, "Eliminating the effects of motion during radiofrequency lesion delivery using a novel contact-force controller." (2019). Medical Biophysics Publications. 92.

https://ir.lib.uwo.ca/biophysicspub/92 


\section{Authors}

Daniel Gelman, Allan C Skanes, Douglas L Jones, Michael Timofeyev, Tal Bar-On, and Maria Drangova 


\section{Eliminating the effects of motion during radiofrequency lesion}

\section{delivery using a novel contact force controller}

Daniel Gelman, BEng, ${ }^{\text {a,b }}$ Allan C. Skanes, MD, FHRS, ${ }^{\mathrm{c}}$ Douglas L. Jones, PhD, ${ }^{\mathrm{d}}$ Michael Timofeyev, Tal Baron, ${ }^{\mathrm{e}}$ and Maria Drangova, $\mathrm{PhD}^{\mathrm{a}, \mathrm{b}, \mathrm{f}}$

${ }^{a}$ Robarts Research Institute, Schulich School of Medicine and Dentistry, The University of Western Ontario, London, Canada

${ }^{\mathrm{b}}$ School of Biomedical Engineering, The University of Western Ontario, London, Canada

${ }^{\mathrm{c}}$ Department of Medicine, Schulich School of Medicine and Dentistry, The University of Western Ontario, London, Canada

${ }^{\mathrm{d} D e p a r t m e n t}$ of Physiology and Pharmacology, Schulich School of Medicine and Dentistry, The University of Western Ontario, London, Canada

${ }^{\mathrm{e}}$ Biosense Webster, Haifa, Israel

${ }^{\mathrm{f}}$ Department of Medical Biophysics, Schulich School of Medicine and Dentistry, The University of Western Ontario, London, Canada

Funding Sources: This work was supported in part by the Canadian Institutes of Health Research, the Ontario Research Fund, and the Cardiac Arrhythmia Network of Canada.

Corresponding Author: Maria Drangova, Ph.D., Robarts Research Institute, Western University, 1151 Richmond St. N., London, ON, Canada, N6A 5K8, (519) 931-5777 Ext. 24064. E-mail: mdrangova@robarts.ca.

Keywords: contact force; contact-force control; catheter ablation; atrial fibrillation; arrhythmia

Author Contributions: DG: concept/design, data collection, data analysis, statistics, drafting article, approval of article; ACS: data collection, critical revision of article, approval of article; DLJ: data collection, critical revision of article, approval of article; MT: design/concept, approval of article; TB: concept, approval of article; MD: Funding secured by, data collection, data analysis, statistics, critical revision of article, approval of article.

Disclosure: Biosense Webster has provided equipment on loan. D.G., A.C.S., and M.D. have applied for a patent relating to the contact-force controller. The technology has been licensed to Aufero Medical, owned in part by D.G., A.C.S., and M.D. A.C.S. receives consulting and research grants from Biosense Webster. 


\begin{abstract}
Introduction: Catheter-tissue contact force is a determinant of RF ablation lesion effectiveness. However, ablation on a beating heart is subject to force variability, making it difficult to optimally deliver consistently durable and transmural lesions. This work evaluates improvements in contact force stability and lesion reproducibility by using a Catheter Contact-Force Controller (CFC) during lesion delivery in vitro and in vivo.
\end{abstract}

Methods and Results: Using a sheath and force-sensing catheter, an experienced operator attempted to maintain a constant force of $20 \mathrm{~g}$ at targets within the LA, RA, and LV of a pig manually and using the $\mathrm{CFC}$; the average force and contact-force variation (CFV) achieved using each approach were compared. Ablation lesions ( $20 \mathrm{~W}, 30$ seconds, $17 \mathrm{ml} / \mathrm{min}$ irrigation) were created in bovine tissue samples mounted on a platform programmed to reproduce clinically relevant motion. CFC-assisted lesions were delivered to stationary and moving tissue with forces of 5 to $35 \mathrm{~g}$. Mimicking manual intervention, lesions were also delivered to moving tissue while the CFC was disabled. Resultant lesion volumes were compared using two-way ANOVA. When using the $\mathrm{CFC}$, the average force was within $1 \mathrm{~g}$ of the set level, with a CFV less than $5 \mathrm{~g}$, during both in vitro and in vivo experiments. Reproducible and statistically identical $(\mathrm{p}=0.82)$ lesion volumes proportional to the set force were achieved in both stationary and moving tissue when the CFC was used.

Conclusions: CFC assistance maintains constant force in vivo and removes effect of motion on lesion volume during RF lesion delivery. 


\section{Introduction}

Catheter-tissue contact force is a major determinant of catheter ablation lesion size uncertainty during radiofrequency (RF) power application. ${ }^{1-5}$ Insufficient contact force results in inadequate lesion production and increased AF recurrence rates, ${ }^{6-8}$ while excessive contact force is associated with tissue over-heating, increased incidence of steam pops, perforation, thrombus formation, and collateral tissue damage to the esophagus and phrenic nerve. ${ }^{2,-13}$ In addition to the average force, contact-force variability has been shown to be an essential parameter in determining RF lesion formation. ${ }^{14}$ Improving contact-force stability enables RF power to be effectively deposited within the tissue and not the surrounding blood pool, and this, in turn, could be critical to achieving long-term success. ${ }^{15}$

A recent meta-analysis, summarizing all randomized controlled trials investigating contact force in $\mathrm{AF}$ ablation, reports that contact force variability, rather than average force alone, has emerged as a critical element that governs lesion size and ablation outcomes. ${ }^{16}$ The EFFICAS II trial demonstrated durable PVI using a contact-force target of $20 \mathrm{~g}$, with a narrow range between $10 \mathrm{~g}$ and $30 \mathrm{~g} .{ }^{4}$ Similarly, the SMART-AF trial showed an increase in clinical success when more time was spent within a pre-defined force range (i.e. reduced force variability) ${ }^{15}$ operators who stayed within a selected range for more than $80 \%$ of the time during RF applications were more than 4 times more likely to have clinical success in comparison to those who did not. In further studies, Ullah et al. ${ }^{17}$ showed that, for the same accumulated FTI, RF ablation lesions delivered with contact-force variation less than $5 \mathrm{~g}$ significantly increased impedance drops and achieved higher maximums compared to lesions with greater variation. ${ }^{17}$ The authors of this study suggest that improving force stability rather than merely delivering more FTI can produce more effective lesions. In a similar study, Makimoto et $a l .{ }^{18}$ found that a relative standard deviation less than $30 \%$ of the mean force (e.g. $10 \pm 3 \mathrm{~g}$ or $20 \pm 6 \mathrm{~g}$ ) correlated with a reduction in reconnection gaps during PVI. ${ }^{18}$ Both of these studies identified regional locations typically associated with AF reconnection, such as the LA roof, with parameters demonstrating high contact-force variability.

Despite this evidence, in clinical practice, it is difficult to maintain sufficient average contact force and reduce contact-force variability reliably. ${ }^{18-22}$ The adjustments made by an operator are limited by human factors (perception and reaction time) and system delays (including time required to average and display a mean force level), making it impossible to compensate for force variability in real-time and highlighting the clear need for new technology to control catheter-tissue contact force and optimize catheter ablation techniques.

In our previous work, ${ }^{23}$ we developed and evaluated a catheter contact-force controller (CFC) that monitors catheter-tissue contact force in real-time and simultaneously adjusts the position of the ablation catheter within a sheath to compensate for variations in contact force that occur due to cardiorespiratory motion. Preliminary lab-bench tests demonstrated that using the CFC resulted in significant improvement in the stability and control of catheter-tissue contact force on moving tissuemimicking material.

The aim of the present study was to demonstrate the ability of the CFC to maintain a set level of force in vivo and determine the device's impact on lesion production when compared to manual intervention. In the first part of the study, the device was tested in a porcine model in vivo, where an experienced electrophysiologist attempted to maintain a set force level with and without the assistance of the CFC. In the second part of the study, an in vitro contractile bench model tested the hypothesis that the CFC helps deliver prescribed and reproducible ablation lesions despite the presence of clinically relevant tissue motion under fixed conditions of RF power and duration. In these experiments, the sizes of lesions delivered to moving tissue samples with and without the CFC were compared to lesions delivered to stationary tissue. 


\section{Catheter Contact-Force Controller}

\section{Methods}

The CFC is a simple add-on to a conventional force-sensing catheter used in conjunction with a steerable sheath, described in detail in Gelman et al. ${ }^{23}$ Briefly, the CFC actuation unit is clamped onto the back-end of a steerable sheath near the hemostatic seal (Figure 1); a linear motor clamped onto the catheter shaft via a locking adaptor adjusts catheter motion with respect to the sheath. The CFC's embedded electronics monitor the contact force in real-time and use 1 of 2 control algorithms to adjust the catheter position within the sheath to maintain constant force at the catheter tip.

First, a model-based adaptive control system ${ }^{24}$ was implemented to assist in cases where gradual changes in contact force are required (e.g. respiratory motion). However, such adaptive algorithms perform poorly in the presence of large, rapid fluctuations of contact force, such as those caused by systolo-diastolic motion. To address this, an additional control system, which uses repetitive control, ${ }^{25}$ was implemented. Specifically, the repetitive control system uses the heart rate, derived from the ECG, as a priori information to improve disturbance-rejection performance during the presence of fast-moving periodic cardiac motion. In the current implementation, the user selected the control mode (adaptive versus repetitive) based on the observed contact-force profile.

\section{Force Control Performance In Vivo}

Animal studies were performed in accordance with institutional and national guidelines and approved by the University of Western Ontario Animal Use and Care Committee (Protocol \#2013-064). Two male farm pigs weighing approximately $35 \mathrm{~kg}$ were used for the study.

Using standard procedures, each pig was anesthetized and prepared for catheterization. ${ }^{26}$ Each pig was intubated, and the ventilation rate was set for 20 BPM. A research-based catheter mapping system (CARTO 3, Biosense Webster) provided real-time force information. A mobile C-arm fluoroscopic x-ray unit (OEC 9900 Elite, General Electric) and intracardiac echocardiography system (Acuson Sequoia, Seimens) were used to assist catheter navigation (Figure 2). A steerable sheath (Agilis NxT, Abbott Laboratories) and a force-sensing ablation catheter (SMARTTOUCH, Biosense Webster) with the CFC attached were inserted into the heart via right femoral access. A pacemaker attached to an external generator was introduced via the right jugular vein and placed in the superior RA to provide cardiac pacing at 115 BPM throughout the experiment. A shaped transseptal needle (Brockenbrough, Medtronic) was introduced via the left femoral vein and used to gain access to the left side of the heart, under the guidance of an intracardiac echocardiography catheter (Acuson AcuNav, Seimens).

A skilled electrophysiologist (A.C.S.) manipulated the catheter and steerable sheath to target locations in the LA, RA and LV. At each location, the physician attempted to maintain $20 \mathrm{~g}$ for 30 seconds with conventional manual intervention. The CFC was then enabled and set to maintain the force at $20 \mathrm{~g}$. For all interventions, the catheter tip was maintained perpendicular to the surface of the tissue to mimic ideal ablation conditions and maximize motion-related force variability; a cathetertissue incidence angle of between $0^{\circ}$ and $45^{\circ}$ from normal was considered perpendicular, as defined by Ullah et al. ${ }^{17}$ It is important to note that the anatomy of the pig LA is quite different from the human. Most importantly, the PV region is often a confluence with minimal musculature. Also, the LA appendage is very large and makes up a large portion of the surface area. Sites in the LA were targeted as follows; after transseptal access was obtained the catheter was advanced to the LA appendage, withdrawn to the LA roof and turned posteriorly to the posterior LA wall to allow sampling of several sites within the LA.

Contact-force profiles of manual and CFC-assisted interventions were recorded and compared. The average contact force and variation were calculated for manual and contact-force-controlled experiments. Variability in contact force and was calculated as contact-force variation $(\mathrm{CFV})^{17}-$ i.e.

Preprint of Gelman D, Skanes AC, Jones DL, Timofeyev M, Bar-On T, Drangova M. Eliminating the effects 4 of motion during radiofrequency lesion delivery using a novel contact-force controller;

J Cardiovasc Electrophysiol. 2019 Sep;30(9):1652-1662. doi: 10.1111/jce.14093. Epub 2019 Aug 7. 
the difference between the mean trough and peak forces - as well as the relative standard deviation (RSD) or the coefficient of variation. ${ }^{18}$ Calculations were performed using a custom script in MATLAB (MathWorks).

Several CFC-assisted ablations were also delivered within the RA and LA to ensure the delivery of power did not affect the performance of the CFC. Visual inspection of the lesions after the pigs were euthanized was performed only to confirm that power was indeed delivered.

\section{Lesion Formation Under Contact-Force Control: In Vitro Setup}

The impact of the CFC on lesion production was evaluated using an in vitro model simulating the beating heart, adapted from the experimental setup and protocol of Shah et al. ${ }^{14}$ Illustrated in Figure 3, the distal portion of a steerable sheath, housing a force-sensing ablation catheter, was deflected $90^{\circ}$ and mounted over a Delrin platform submerged within an acrylic tank filled with $0.7 \%$ saline at room temperature. The platform was fixed to a custom-built linear motion stage, which could be raised and lowered by a gear motor. A generator (EP Shuttle, Stockert GmbH) in power-control mode delivered RF energy between the catheter-tip electrode and a ground plate fixed underneath the platform. Slabs of bovine muscle, $20-30 \mathrm{~mm}$ thick, were placed on the platform. A peristaltic pump (CoolFlow, Biosense Webster) connected to the irrigation port of the catheter delivered $0.7 \%$ saline during RF delivery.

The linear motion phantom was driven in 1 of 2 modes, either reproducing contact-force measurements recorded by force-sensing catheters during RF delivery in patients, ${ }^{25}$ or simulating empirically derived cardiorespiratory motion profiles (Figure 4). For the simulated motion profiles, the respiratory component resembled typical tidal lung volume profiles, while the cardiac component replicated either intermittent or variable contact profiles. Simulated cardiac and respiratory motion waveforms with user-defined amplitudes and frequencies were used to reproduce scenarios of intermittent catheter-tissue contact, induced patient apnea, and various combinations of dominant respiratory or cardiac motion. Simulated motion was used to isolate tissue motion, without the introduction of artifacts potentially introduced by a physician operation or the force sensor itself (i.e. noise).

\section{Lesion Formation Under Contact-Force Control: Protocol}

RF energy was delivered to the tissue at $20 \mathrm{~W}$ for 30 seconds; the irrigation flow rate was 17 $\mathrm{ml} / \mathrm{min}$. First, CFC-assisted ablation lesions were delivered to stationary tissue at 4 force levels $(5 \mathrm{~g}$, $15 \mathrm{~g}, 25 \mathrm{~g}$, and $35 \mathrm{~g}$ ). These lesions were used as "control lesions" - i.e. created in the absence of motion. The same set of ablation protocols was then repeated, but the motion stage was set to execute randomly selected motion profiles with the CFC enabled to maintain the force constant at 1 of the 4 force levels. Lastly, to mimic lesion production during manual intervention, lesions were delivered to moving tissue while the CFC was disabled. For all experimental conditions, contact-force profiles were recorded, and the mean, CFV and RSD were calculated.

\section{Lesion Formation Under Contact-Force Control: Lesion Measurement}

Once lesions were delivered, the tissue slabs were sliced along the center of each lesion using a scalpel to expose the cross-section. Photographs of sectioned lesions were taken with a camera mounted in a 3D-printed custom bracket to ensure that the cross-section of the lesion was centered and parallel to the aperture of the camera; a constant distance between the camera and lesion surface enabled calibration of the images. The photographs were randomized prior to analysis by a blinded observer. The dimensions of the necrotic zone within each lesion were measured using digital calipers in the image-analysis software ImageJ. ${ }^{27}$ The maximum diameter, maximum depth, and depth at the

Preprint of Gelman D, Skanes AC, Jones DL, Timofeyev M, Bar-On T, Drangova M. Eliminating the effects 5 of motion during radiofrequency lesion delivery using a novel contact-force controller;

J Cardiovasc Electrophysiol. 2019 Sep;30(9):1652-1662. doi: 10.1111/jce.14093. Epub 2019 Aug 7. 
maximum diameter for each lesion were measured (Figure S1). Lesion volumes were calculated using the formula for a truncated oblate spheroid:

$$
V_{\text {Lesion }}=V_{\text {Spheroid }}-V_{\text {Spheroidal Cap }}=\frac{4 \pi a^{2} c}{3}-\frac{\pi a^{2} h^{2}(3 c-h)}{3 c^{2}},
$$

where $a=$ Maximum Diameter $/ 2, c=$ Maximum Depth - Depth at Maximum Diameter, and $h=c$-Depth at Maximum Diameter. Similar ellipsoidal modelling of lesion volume has been used previously. 14

\section{Lesion Formation Under Contact-Force Control: Statistical Analysis}

Statistical analysis was performed using Prism 7 (GraphPad Software, USA). Two-way analysis of variance (ANOVA) was performed for both lesion volume and lesion depth to determine if the set-force level effect on lesion size was statistically significant. Means at each set force level were compared and the Sidak multiple comparison test was used to test the hypothesis that the volume and depth of CFC-controlled lesions delivered to moving tissue do not differ from those of lesions delivered to stationary tissue. A p-value of less than 0.05 was considered statistically significant.

\section{In Vivo Experiments}

\section{Results}

Fourteen contact-force-control experiments were performed: 8 in the LA, 5 in the RA and 1 in the LV. Of these, 12 experiments were performed with the CFC set to repetitive control mode using the heart rate determined from ECG and 2 with the CFC set for adaptive control mode (the results below are averages of all 14 experiments). Representative examples of the contact force profiles achieved when using repetitive control and adaptive control are provided in Figure 5A-5C and Figure $5 \mathrm{D}$, respectively, with Video S1 demonstrating a real-time representation of force control. Through manual catheter intervention, the interventionalist was able to maintain an average force level of $24.6 \pm$ $6.4 \mathrm{~g}$, which was larger than the target of $20 \mathrm{~g}$; the smallest average force was $14.9 \mathrm{~g}$, and the largest average was $30.5 \mathrm{~g}$. In contrast, for all CFC-assisted interventions, with a set force of $20 \mathrm{~g}$, the average contact force was $19.9 \pm 0.3 \mathrm{~g}$ [range $18.9 \mathrm{~g}$ to $20.1 \mathrm{~g}$ ]. When compared to manual intervention, CFCassistance resulted in a reduction in CFV from $31.6 \pm 15.9 \mathrm{~g}$ to $4.6 \pm 1.1 \mathrm{~g}(\mathrm{p}<0.0001)$ and RSD from $53 \pm 24 \%$ to $20 \pm 7 \%(\mathrm{p}=0.005)$.

Analyzing CFC-assisted performance on a regional level, no significant difference in CFV between the LA $(4.9 \pm 1.2 \mathrm{~g}, \mathrm{n}=8)$ and RA $(4.0 \pm 0.6 \mathrm{~g}, \mathrm{n}=5)$ was observed $(\mathrm{p}=0.161)$. While testing the CFC in the LV, engaging the CFC induced premature ventricular contractions (PVC) in all but one attempt. The PVCs caused the repetitive control system to temporally lose synchrony with the cardiac motion of the pig heart, resulting in poor performance. In the single case where PVCs were not induced (Figure S2C), the CFV was reduced from $32.9 \mathrm{~g}$ to $8.6 \mathrm{~g}$ when the CFC was engaged. Importantly, the maximum force measured did not exceed $50 \mathrm{~g}$ in any of the cases when the CFC was engaged (all three chambers) and was lower than the maximum measured during manual intervention.

\section{Contact-Force Control In Vitro}

The mean, CFV and RSD of the contact-force profiles recorded during CFC-assisted ablationlesion delivery for the 3 sets of experiments (CFC-assisted lesion delivery to stationary tissue, CFCassisted lesion delivery to moving tissue, and manual lesion delivery to moving tissue) are shown in Table 1. Similar to the in vivo results, the average contact force remained within $1 \mathrm{~g}$ of the set level, and the CFV reduced to less than $5 \mathrm{~g}$ on average for all set levels of the CFC. Specifically, CFCassisted intervention reduced CFV from $19.4 \pm 10.2 \mathrm{~g}$ to $4.0 \pm 1.0 \mathrm{~g}(\mathrm{p}<0.0001)$ and RSD from $64 \pm$ $39 \%$ to $25 \pm 14 \%(\mathrm{p}<0.0001)$. When setting the CFC with a modest set force $(15 \mathrm{~g}$ and greater $)$, the 
resulting RSD improved to $17 \pm 7 \%$. Versus manual intervention, employing the CFC narrowed the distribution of contact forces and centered in on the set force, improving precision and accuracy. Furthermore, the range (the difference between maximum and minimum) of contact forces was reduced by at least a factor of 2 . It is important to note that these results include several ablation experiments performed while the motion phantom was driven to reproduce variability that resembles patient, catheter, or unpredictable cardiorespiratory motion. Even in these irregular motion cases, CFV was reduced to less than $5 \mathrm{~g}$ (and RSD to below 30\%) when the CFC was used. Comparative example force profiles achieved with the CFC when the motion was irregular are shown in Figure 6.

\section{Lesion Production With and Without Force Control}

Representative example photographs of cross-sections of delivered lesions are provided in Figure 7. The measured depths and calculated volumes of the CFC-assisted ablation lesions on stationary and moving tissue, as well as lesions delivered to mimic manual intervention (without the CFC), are presented in Figure 8. As determined by two-way ANOVA, statistically significant differences were observed between lesion depth and volume for lesions delivered to stationary or moving tissues at different set force levels $(\mathrm{p}<0.0001)$. Most importantly, while employing the CFC, motion during lesion delivery had no effect on lesion depth or volume ( $p=0.82$ and 0.78 , respectively) compared to stationary lesions. On the other hand, manual intervention delivered to moving tissue resulted in highly inconsistent lesion depth and volume. Multiple comparison analysis revealed a small difference in lesion depth (not volume) between stationary and moving tissue when the set level was 5 g.

\section{Discussion}

In this study we demonstrated that: a) on average, the CFC can consistently control force to within $1 \mathrm{~g}$ of the set level; b) the variation in contact force dropped below $5 \mathrm{~g}$ when using the CFC - a 5-fold improvement over manual intervention; c) when using the CFC, the catheter tip never lost contact with the tissue and never approached dangerous force levels; d) delivered ablation lesions were reproducible regardless of myocardial motion, and finally, e) delivered lesions to moving tissue were of the same depth and volume as lesions delivered in the absence of tissue motion. Recalling the clinical studies performed by Ullah et al. ${ }^{17}$ and Makimoto et al. ${ }^{18}$, which respectively report that a CFV of less than $5 \mathrm{~g}$ ensures a maximum impedance drop and RSD less than $30 \%$ is correlated with more durable lesions, our results suggest that using the CFC can assist clinicians in achieving these previously unattainable goals.

During the in vitro ablation experiments, on average a 2-fold reduction was observed between the standard deviations of lesion volume (and depth) of CFC-assisted and manually delivered lesions. This indicates improved precision and accuracy in lesion production over manual intervention while the tissue was moving. More importantly, regardless of tissue motion, lesions delivered were reproducible and statistically identical in size compared to lesions delivered to stationary tissue, indicating that lesions were delivered to moving tissue as if no motion was present. The slightly significant difference in lesion depth noted at the 5-g level is attributed to the likelihood that at low force levels, lesion production is more vulnerable to variation in force - improving the control performance of the CFC may prevent this.

During in vivo evaluation in the porcine model, the CFC demonstrated the ability to compensate for significant myocardial motion, greater than that observed in humans. The CFC was capable of reducing spikes of $50 \mathrm{~g}$ caused by the systolo-diastolic motion of the heart at rates over 110 $\mathrm{BPM}$ to negligible disturbances (Figure 5A and 5B). This performance was achieved in all target locations in the LA and RA. However, in the LV, we observed poorer performance, likely due to the occurrences of PVCs and the fact that the repetitive controller was optimized for atrial use. Further 
development of the CFC control system, which may involve fine-tuning of the repetitive controller or utilizing the adaptive controller, is required before a more extensive evaluation in the LV.

A primary concern of using the CFC is the possibility of perforating the tissue while the CFC is engaged. For this reason, the CFC is programmed to continuously monitor contact force and automatically retract the catheter back into the sheath and disable the force control algorithm if the force level exceeds a user-defined limit (currently set to $80 \mathrm{~g}$ ). Furthermore, it is important to note that during all CFC-interventions performed in vivo and in vitro, the force never exceeded $50 \mathrm{~g}$. We also demonstrated, in our first study, ${ }^{23}$ that the CFC has shown to effectively react to large and sudden changes in tissue displacement that would otherwise result in large spikes of contact force and potentially cause tissue damage.

The development of two different control systems (adaptive and repetitive) was required to handle types of motion typically observed during patient therapy. Different controllers were required to compensate for the delay, sampling rate and noise characteristics of the force sensor incorporated on the catheter tip. The repetitive control system can be used to eliminate fast systolo-diastolic force variations when they are the most dominant component, but strictly requires that tissue motion is periodic (i.e. normal sinus rhythm). The current version of the repetitive control system is unable to effectively handle the non-periodic disturbances observed in patients in AF or instances of mechanically-induced PVCs but was able to compensate for motion associated with heart-rate drifts up to $\pm 7 \%$. On the other hand, the adaptive control system ignores fast disturbances caused by cardiac motion and can only reject gradual changes in force variations and is suitable for most interventions where the largest motions are caused by respiration or involuntary movement. The adaptive controller may, therefore, be ideally suited for use during arrhythmias, where cardiac-induced motion is expected to be smaller. Lastly, in the cases where both cardiac and respiratory motion is large, both control algorithms will have reduced benefit; however, such cases are rare.

The benefit of the CFC is dependent on the angle between the catheter tip and the surface of the tissue, with the highest improvement achieved during perpendicular contact, when the effect of motion is greatest. On the other hand, when the catheter is nearly parallel to the tissue, such as during CTI ablation, the CFC is expected to have little benefit. In this study, the CFC was evaluated at angles up to $45^{\circ}$; however, a significant reduction of force variability has been observed at angles as large $60^{\circ}$ from the normal (data not shown). Additional studies are required to determine a threshold angle beyond which using the CFC will not provide benefit.

Systolo-diastolic cardiac motion and respiration are the primary contributors to variability in contact force. ${ }^{1,21,28}$ Clinical techniques used to mitigate their effect include rapid atrial pacing, highfrequency jet ventilation (HFJV), and induced apnea. ${ }^{28-33}$ Rapid pacing has been shown to reduce the standard deviation in force by less than $1 \mathrm{~g}$ compared to its non-paced counterpart, and its effectiveness is dependent on regional anatomical location within the LA. ${ }^{29}$ In a study examining almost 30,000 RF applications, HFJV only reduced force variability index by 0.06 on average in comparison to normal ventilation. ${ }^{33}$ In contrast, to achieve optimal force variability based on the variability criteria of CFV (less than $5 \mathrm{~g}^{17}$ ) and RSD (less than $30 \%{ }^{18}$ ), the CFC reduced standard deviation in the force profiles by $6.3 \mathrm{~g}$ on average and the force variability index by 0.21 . Importantly, the CFC is a device that can be used independently to compensate for the effect of motion or to supplement one of these techniques. In particular, the combination of induced apnea and the CFC working in repetitive-control mode would be an extremely effective way to control force (Figure 5B).

Emerging catheter ablation techniques in treating AF include delivering RF energy with highpower (typically 70-90 W) for 4-8 seconds. In preclinical porcine studies, high-power short-duration (HP-SD) ablation have been shown to reduce the effects of lesion-to-lesion variability and catheter instability that cause reversible partial-thickness lesion production. ${ }^{34,35}$ To date, no results have been reported evaluating the role of contact-force variability in lesion production when HP-SD ablation is 
used. However, HP-SD PVI studies in swine have demonstrated that contact force values between 10 and $20 \mathrm{~g}$ resulted in successful ablation, ${ }^{34}$ while fluctuating forces exceeding $40 \mathrm{~g}$ caused steam pops. ${ }^{35}$ For clinical applications of HP-SD, electrophysiologists would need to maintain contact force within the guidelines set out by these studies, which may be difficult to achieve reproducibly. These preclinical studies indicate the importance of controlling contact force and minimizing variability, regardless of the method of RF delivery.

\section{Study Limitations}

While the study demonstrates that the CFC can reduce the effects of motion on contact force during ablations in vivo and in vitro in a limited set of experiments, more comprehensive studies of the device are required (e.g. in the LV) before the real impact of using the CFC can be determined.

Typical contact-force profiles observed in the clinical setting are dominated by respiration; contact-force disturbances caused by cardiac motion are often negligible in most targets. Surprisingly, the opposite relationship was observed in the porcine model, where for most target locations, disturbances were dominated by systolo-diastolic cardiac motion, while disturbances caused by respiration were smaller in comparison. As a result, the primary control mode that was tested in vivo was the repetitive control system. While the adaptive control system could not be extensively evaluated in vivo, this control mode was thoroughly tested in the in vitro experiments.

Lesion production in the in vitro study does not directly reflect lesion production in humans. Our experimental set up did not mimic blood and tissue temperature and did not model convective and perfusive cooling. However, all experimental conditions remained consistent from lesion-to-lesion, and the results generated from these experiments were solely dependent on tissue motion and contact force. The measured reference impedance (75-85 $\Omega$, achieved by titrating the salinity to $0.7 \%$ ) was low compared to clinical levels but was chosen to enable the generation of measurable lesions at clinically relevant contact-force levels and duration. Finally, lesion volume was measured only by a single observer and was based on measurements of the necrotic area. Systematic bias was avoided by cropping, anonymizing, and randomizing the photographed tissue samples, thereby blinding the observer to the ablation protocol used for each lesion.

\section{Conclusions}

Monitoring catheter-tissue contact force has become an essential tool for RF catheter ablation in patients. Until this work, contact-force information has been strictly used for visual guidance and navigation. The CFC uses the available real-time force information and optimizes lesion production on moving tissue by controlling catheter-tissue contact force. In this study, we demonstrated the ability for the CFC to significantly improve catheter-tissue contact-force profiles and their impact on lesion production in moving tissue. The results suggest that the CFC can be a valuable add-on tool available to electrophysiologists to optimize lesion delivery techniques and ultimately improve patient outcomes.

\section{Acknowledgements}

The authors thank Amber Parsons, Rachel Daniels, and Matthew Krawecki, at Canadian Surgical Technologies \& Advanced Robotics, for assistance with the animal experiments. We also thank Jeremy Jennison from London Health Sciences Centre's Department of Biomedical Engineering and Arie Gertz, Danielle Dean, and Matt Hendry from Biosense Webster for the continued support of the project. 


\section{References}

1. Nakagawa H, Jackman WM. The Role Of Contact Force In Atrial Fibrillation Ablation. Journal of atrial fibrillation. 2014;7(1):1027.

2. Yokoyama K, Nakagawa H, Shah DC, et al. Novel contact force sensor incorporated in irrigated radiofrequency ablation catheter predicts lesion size and incidence of steam pop and thrombus. Circulation Arrhythmia and electrophysiology. 2008;1(5):354-362.

3. Calkins H, Hindricks G, Cappato R, et al. 2017 HRS/EHRA/ECAS/APHRS/SOLAECE expert consensus statement on catheter and surgical ablation of atrial fibrillation. Heart Rhythm. 2017;14(10):e275-e444.

4. Kautzner J, Neuzil P, Lambert H, et al. EFFICAS II: optimization of catheter contact force improves outcome of pulmonary vein isolation for paroxysmal atrial fibrillation. Europace : 2015;17(8):12291235 .

5. Neuzil P, Reddy VY, Kautzner J, et al. Electrical reconnection after pulmonary vein isolation is contingent on contact force during initial treatment: results from the EFFICAS I study. Circulation Arrhythmia and electrophysiology. 2013;6(2):327-333.

6. Haldar S, Jarman JW, Panikker S, et al. Contact force sensing technology identifies sites of inadequate contact and reduces acute pulmonary vein reconnection: a prospective case control study. Int J Cardiol. 2013;168(2):1160-1166.

7. le Polain de Waroux JB, Weerasooriya R, Anvardeen K, et al. Low contact force and force-time integral predict early recovery and dormant conduction revealed by adenosine after pulmonary vein isolation. Europace. 2015;17(6):877-883.

8. Tofig BJ, Lukac P, Borregaard R, et al. Recurrence after pulmonary vein isolation is associated with low contact force. Scandinavian cardiovascular journal : SCJ. 2018;52(1):28-33.

9. Avitall B, Mughal K, Hare J, Helms R, Krum D. The effects of electrode-tissue contact on radiofrequency lesion generation. Pacing and clinical electrophysiology: PACE. 1997;20(12 Pt 1):28992910.

10. Thiagalingam A, D'Avila A, Foley L, et al. Importance of catheter contact force during irrigated radiofrequency ablation: evaluation in a porcine ex vivo model using a force-sensing catheter. Journal of cardiovascular electrophysiology. 2010;21(7):806-811.

11. Pappone C, Oral H, Santinelli V, et al. Atrio-esophageal fistula as a complication of percutaneous transcatheter ablation of atrial fibrillation. Circulation. 2004;109(22):2724-2726.

12. Shah DC, Namdar M. Real-time contact force measurement: a key parameter for controlling lesion creation with radiofrequency energy. Circulation Arrhythmia and electrophysiology. 2015;8(3):713721.

13. Ikeda A, Nakagawa $\mathrm{H}$, Lambert $\mathrm{H}$, et al. Relationship between catheter contact force and radiofrequency lesion size and incidence of steam pop in the beating canine heart: electrogram amplitude, impedance, and electrode temperature are poor predictors of electrode-tissue contact force and lesion size. Circulation Arrhythmia and electrophysiology. 2014;7(6):1174-1180.

14. Shah DC, Lambert H, Nakagawa H, Langenkamp A, Aeby N, Leo G. Area under the real-time contact force curve (force-time integral) predicts radiofrequency lesion size in an in vitro contractile model. Journal of cardiovascular electrophysiology. 2010;21(9):1038-1043.

15. Natale A, Reddy VY, Monir G, et al. Paroxysmal AF catheter ablation with a contact force sensing catheter: results of the prospective, multicenter SMART-AF trial. J Am Coll Cardiol. 2014;64(7):647656.

16. Ariyarathna N, Kumar S, Thomas SP, Stevenson WG, Michaud GF. Role of Contact Force Sensing in Catheter Ablation of Cardiac Arrhythmias: Evolution or History Repeating Itself? JACC Clinical electrophysiology. 2018;4(6):707-723.

17. Ullah W, Hunter RJ, Baker V, et al. Factors affecting catheter contact in the human left atrium and their impact on ablation efficacy. Journal of cardiovascular electrophysiology. 2015;26(2):129-136.

18. Makimoto H, Tilz RR, Lin T, et al. Incidence and anatomical locations of catheter instability during circumferential pulmonary vein isolation using contact force. International heart journal. 2014;55(3):249-255. 
19. Kumar S, Morton JB, Lee J, et al. Prospective characterization of catheter-tissue contact force at different anatomic sites during antral pulmonary vein isolation. Circulation Arrhythmia and electrophysiology. 2012;5(6):1124-1129.

20. De Ponti R, Marazzi R, Doni LA, Marazzato J, Baratto C, Salerno-Uriarte JA. Optimization of catheter/tissue contact during pulmonary vein isolation: the impact of atrial rhythm. Europace. 2018;20(2):288-294.

21. Sarkozy A, Shah D, Saenen J, et al. Contact force variability during catheter ablation of atrial fibrillation: the role of atrial rhythm and ventricular contractions: co-force AF Study. Circulation Arrhythmia and electrophysiology. 2015;8(6):1342-1350.

22. Matsuda H, Parwani AS, Attanasio P, et al. Atrial rhythm influences catheter tissue contact during radiofrequency catheter ablation of atrial fibrillation: comparison of contact force between sinus rhythm and atrial fibrillation. Heart and vessels. 2016;31(9):1544-1552.

23. Gelman D, Skanes AC, Tavallaei MA, Drangova M. Design and Evaluation of a Catheter Contact-Force Controller for Cardiac Ablation Therapy. IEEE transactions on bio-medical engineering. 2016;63(11):2301-2307.

24. Jain P, Nigam MJ. Design of a Model Reference Adaptive Controller Using Modifited MIT Rule for a Second Order System. Advance in Electronic and Electric Engineering. 2013;3(4):477-484.

25. Qing-Guo W, Han-Qin Z, Yong-Sheng Y, Yong Z, Yu Z. Modified Smith predictor design for periodic disturbance rejection. Paper presented at: Asian Control Conference; 20-23 July 2004.

26. Thakur Y, Jones DL, Skanes A, Yee R, Drangova M. Right-side RF ablation using remote catheter navigation: experimental results in vivo. Journal of cardiovascular electrophysiology. 2012;23(1):8187.

27. Schneider CA, Rasband WS, Eliceiri KW. NIH Image to ImageJ: 25 years of image analysis. Nat Methods. 2012;9(7):671-675.

28. Kumar S, Morton JB, Halloran K, et al. Effect of respiration on catheter-tissue contact force during ablation of atrial arrhythmias. Heart rhythm 2012;9(7):1041-1047.e1041.

29. Aizer A, Cheng AV, Wu PB, et al. Pacing Mediated Heart Rate Acceleration Improves Catheter Stability and Enhances Markers for Lesion Delivery in Human Atria During Atrial Fibrillation Ablation. JACC Clinical electrophysiology. 2018;4(4):483-490.

30. Goode JS, Jr., Taylor RL, Buffington CW, Klain MM, Schwartzman D. High-frequency jet ventilation: utility in posterior left atrial catheter ablation. Heart Rhythm. 2006;3(1):13-19.

31. Elkassabany N, Garcia F, Tschabrunn C, et al. Anesthetic management of patients undergoing pulmonary vein isolation for treatment of atrial fibrillation using high-frequency jet ventilation. Journal of cardiothoracic and vascular anesthesia. 2012;26(3):433-438.

32. Hutchinson MD, Garcia FC, Mandel JE, et al. Efforts to enhance catheter stability improve atrial fibrillation ablation outcome. Heart Rhythm. 2013;10(3):347-353.

33. Sivasambu B, Hakim JB, Barodka V, et al. Initiation of a High-Frequency Jet Ventilation Strategy for Catheter Ablation for Atrial Fibrillation: Safety and Outcomes Data. JACC Clinical electrophysiology. 2018;4(12):1519-1525.

34. Barkagan M, Contreras-Valdes FM, Leshem E, Buxton AE, Nakagawa H, Anter E. High-power and short-duration ablation for pulmonary vein isolation: Safety, efficacy, and long-term durability. Journal of cardiovascular electrophysiology. 2018;29(9):1287-1296.

35. Leshem E, Zilberman I, Tschabrunn CM, et al. High-Power and Short-Duration Ablation for Pulmonary Vein Isolation: Biophysical Characterization. JACC Clinical electrophysiology. 2018;4(4):467-479.

Preprint of Gelman D, Skanes AC, Jones DL, Timofeyev M, Bar-On T, Drangova M. Eliminating the effects 11 of motion during radiofrequency lesion delivery using a novel contact-force controller;

J Cardiovasc Electrophysiol. 2019 Sep;30(9):1652-1662. doi: 10.1111/jce.14093. Epub 2019 Aug 7. 


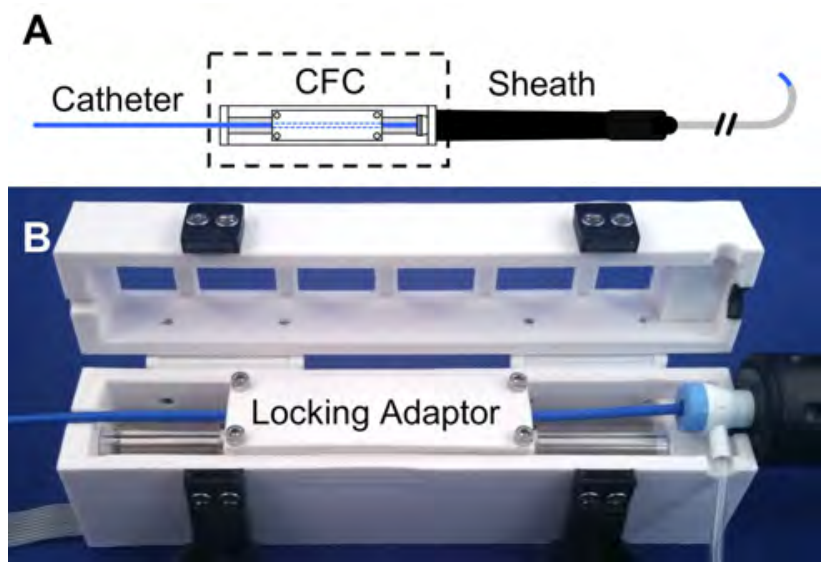

Figure 1. Schematic diagram (A) and photograph (B) of the CFC actuation unit attached to an ablation catheter and steerable sheath. A locking adaptor clamps the CFC to a linear actuator (shown in (B)), which enables the catheter to be displaced axially within the sheath.

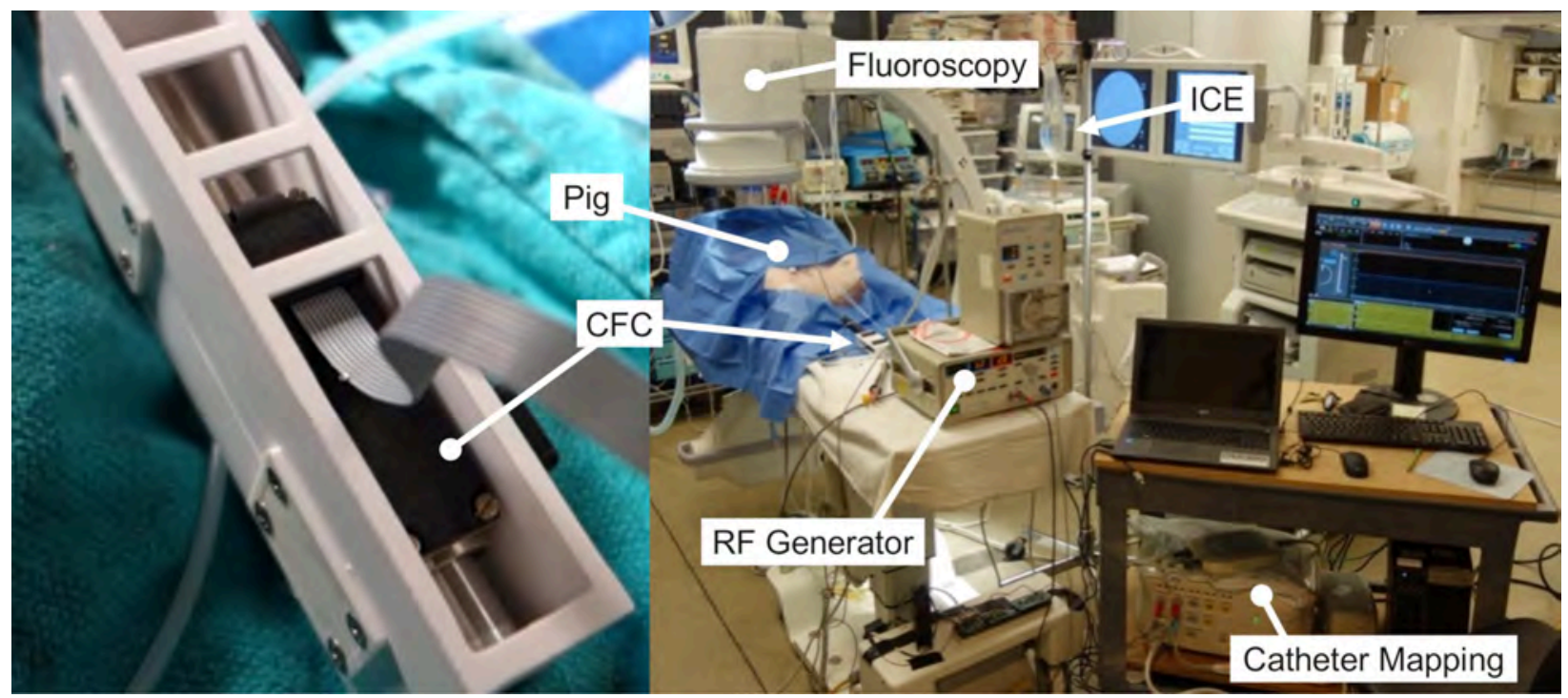

Figure 2. Photograph of the CFC, left, and the in vivo experimental setup, right. 


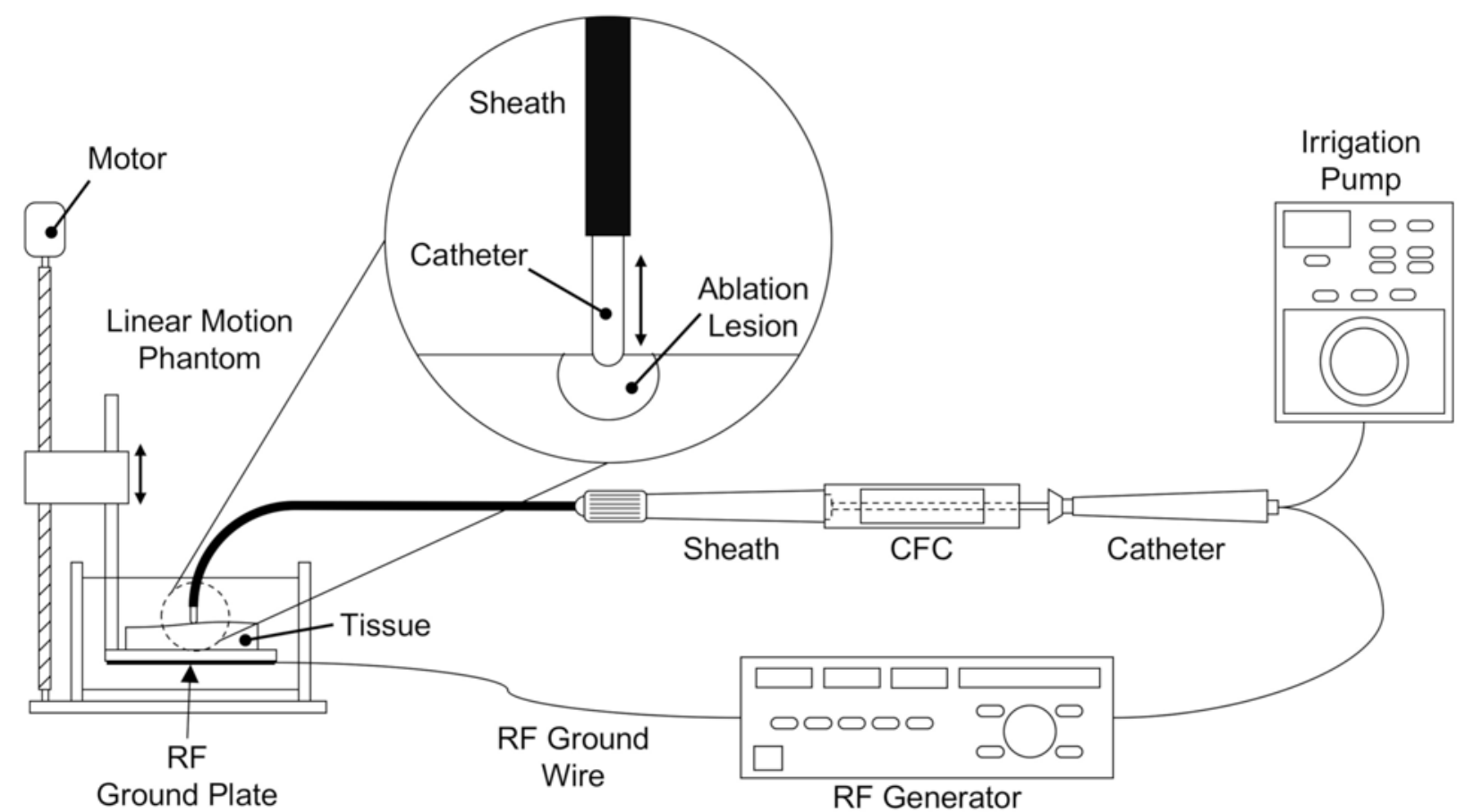

Figure 3. Setup for the in vitro ablation experiments. A linear motion phantom capable of producing clinically relevant myocardial motion moves bovine tissue within a saline bath. The CFC is attached to a force-sensing ablation catheter and steerable sheath, enabling the delivery of RF lesions to moving tissue under force control.
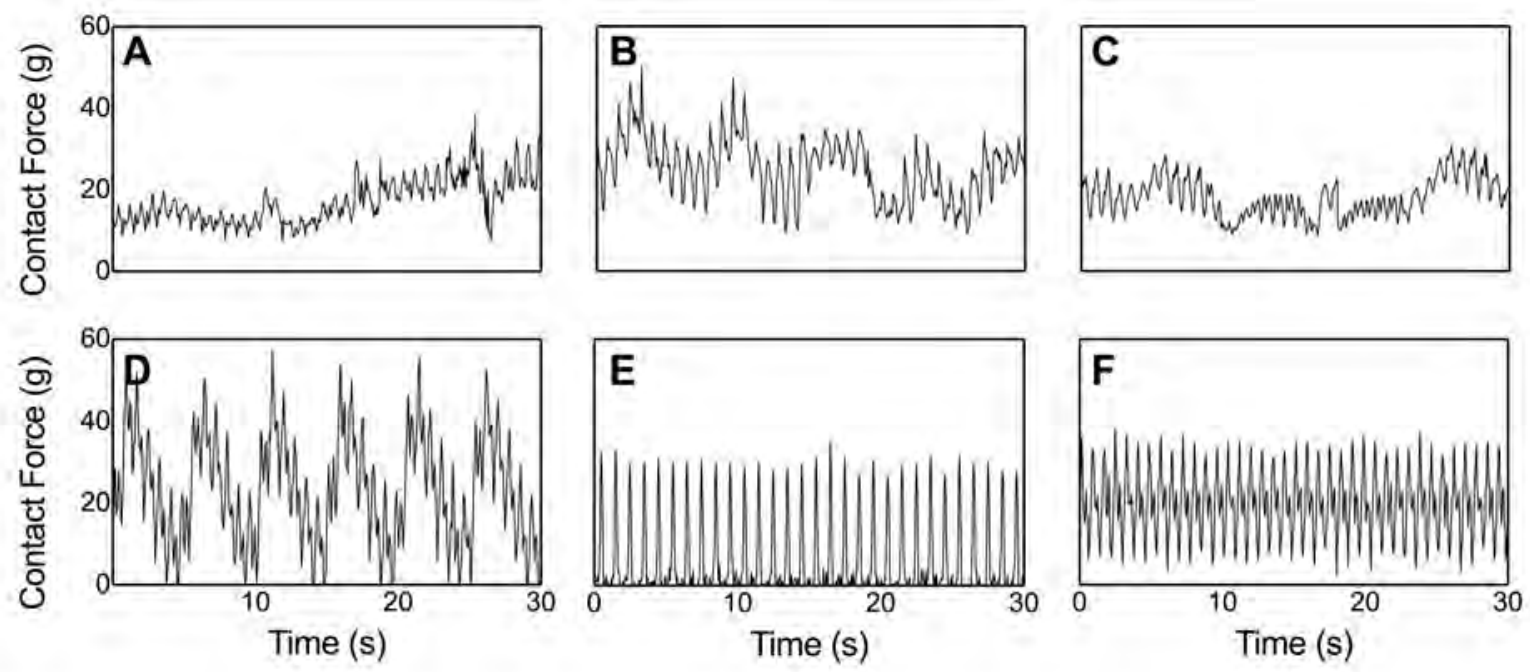

Figure 4. Sample contact-force profiles measured as the linear motion phantom moves tissue against a fixed catheter. The contact-force profiles in (A) trough (C) simulate force generated during patient ablations. The profiles in (D) through (F) represent examples of simulated profiles: (D) combination of cardiac and respiratory motion (cardiac: 75 BPM, $15 \mathrm{~g}$ peak-to-peak; respiration: 12 BPM, 30 g peak-to-peak; offset: $10 \mathrm{~g}$ ), (E) intermittent cardiac motion (cardiac: 75 BPM, 30 g peak-to-peak; respiration: none; offset: none), and (F) variable cardiac motion (cardiac: 75 BPM, 30 g peak-to-peak; respiratory: none; offset: $20 \mathrm{~g}$ ). 

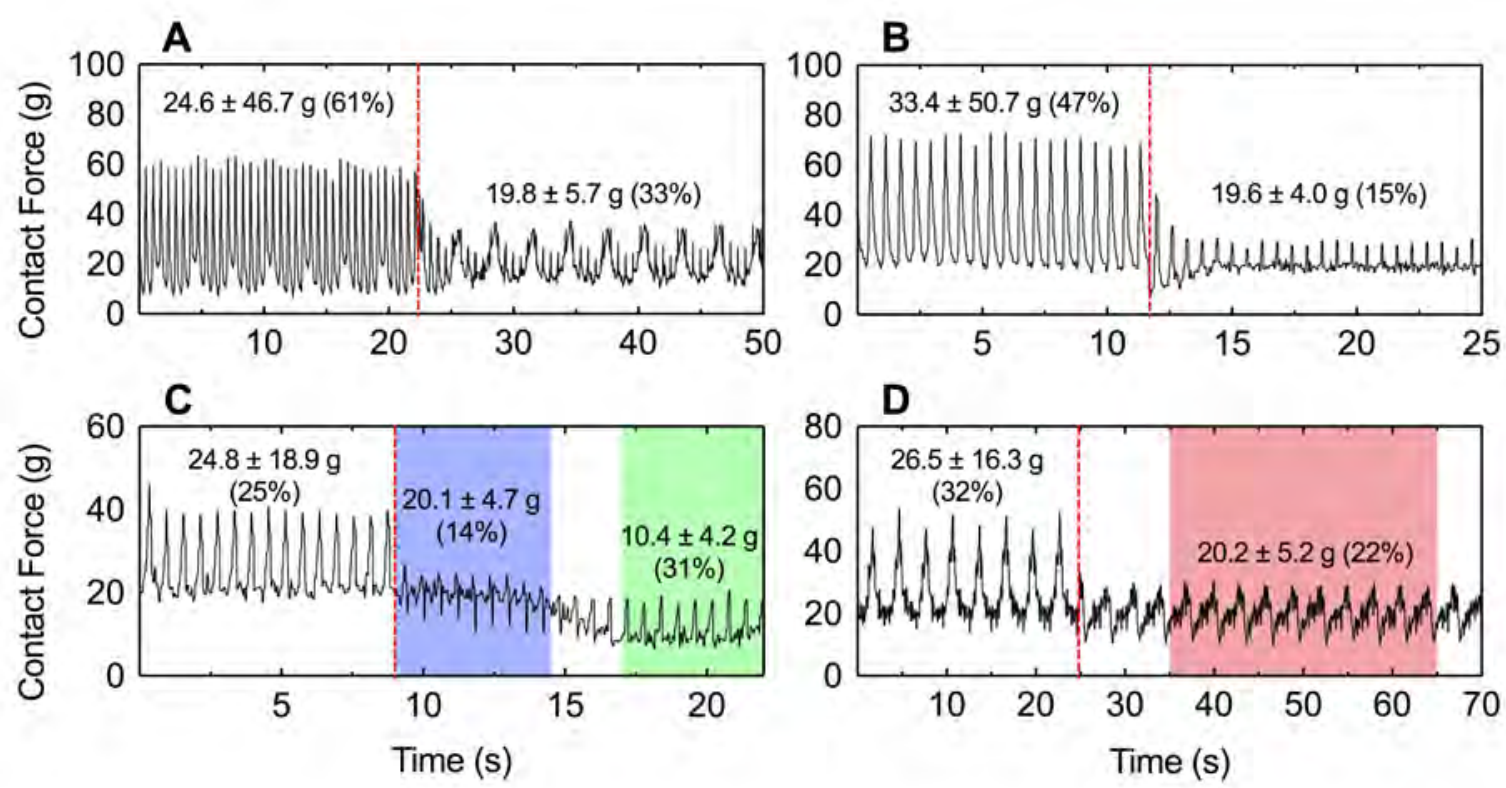

Figure 5. Results from four experiments performed the LA and RA. Each contact-force profile begins with manual intervention, prior to $\mathrm{CFC}$ engagement (red line). The regions targeted were: LAA without (A) and with apnea (B); high RA with apnea (C) while changing the set level of force from $20 \mathrm{~g}$ (blue area) to $10 \mathrm{~g}$ (green area); (D) while delivering a lesion (red area) to the RA septum. The mean contact force $\pm \mathrm{CFV}$ (RSD) during manual and CFC-assisted intervention is reported. Note: the $\mathrm{CFC}$ was able to compensate for myocardial motion greater than that observed in humans (A and B), where the CFC was capable of reducing spikes of over $50 \mathrm{~g}$ at a heart rate of $115 \mathrm{BPM}$ down to negligible disturbances. Note the difference in time and force scales. 

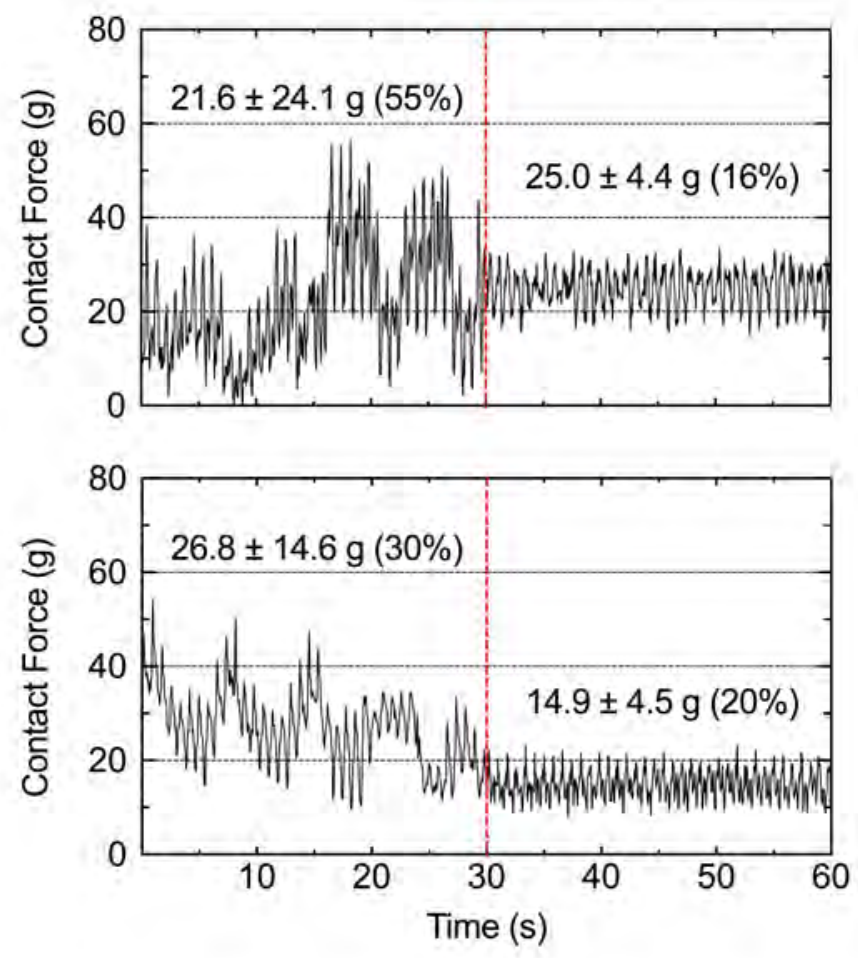

Figure 6. Results from two experiments performed in vitro where the phantom was driven to reproduce motion profiles based on force measurements obtained from force-sensing catheters during RF delivery in patients. The beginning of each experiments shows tissue motion characteristic of unpredictable respiration. With the absence of significant periodic tissue motion, the adaptive control mode of the CFC was engaged (red line) with a set force level of $25 \mathrm{~g}$ (top) and $15 \mathrm{~g}$ (bottom). Average contact force $\pm \mathrm{CFV}$ (RSD) are reported. 


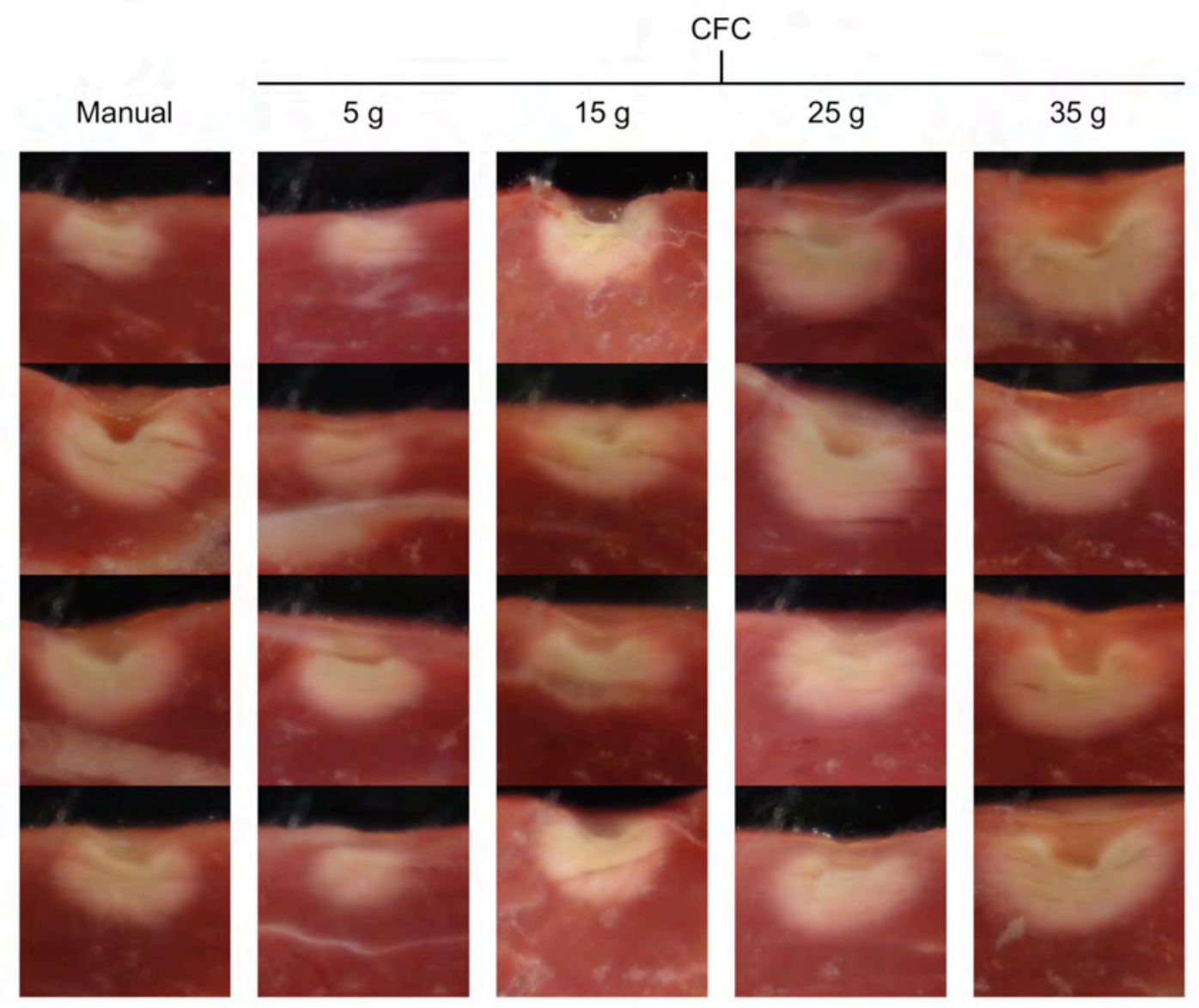

Figure 7. Photographs of the cross-sections of delivered ablation lesions to moving tissue. Representative examples of ablation sizes while the CFC is disabled, representing manual intervention, and while the CFC is set to desired forces of $5 \mathrm{~g}, 15 \mathrm{~g}, 25 \mathrm{~g}$, and $35 \mathrm{~g}$ (organized in columns). Lesions delivered during manual intervention vary in size, while prescribed CFCassisted ablation lesions are precise and reproducible, despite myocardial motion. 

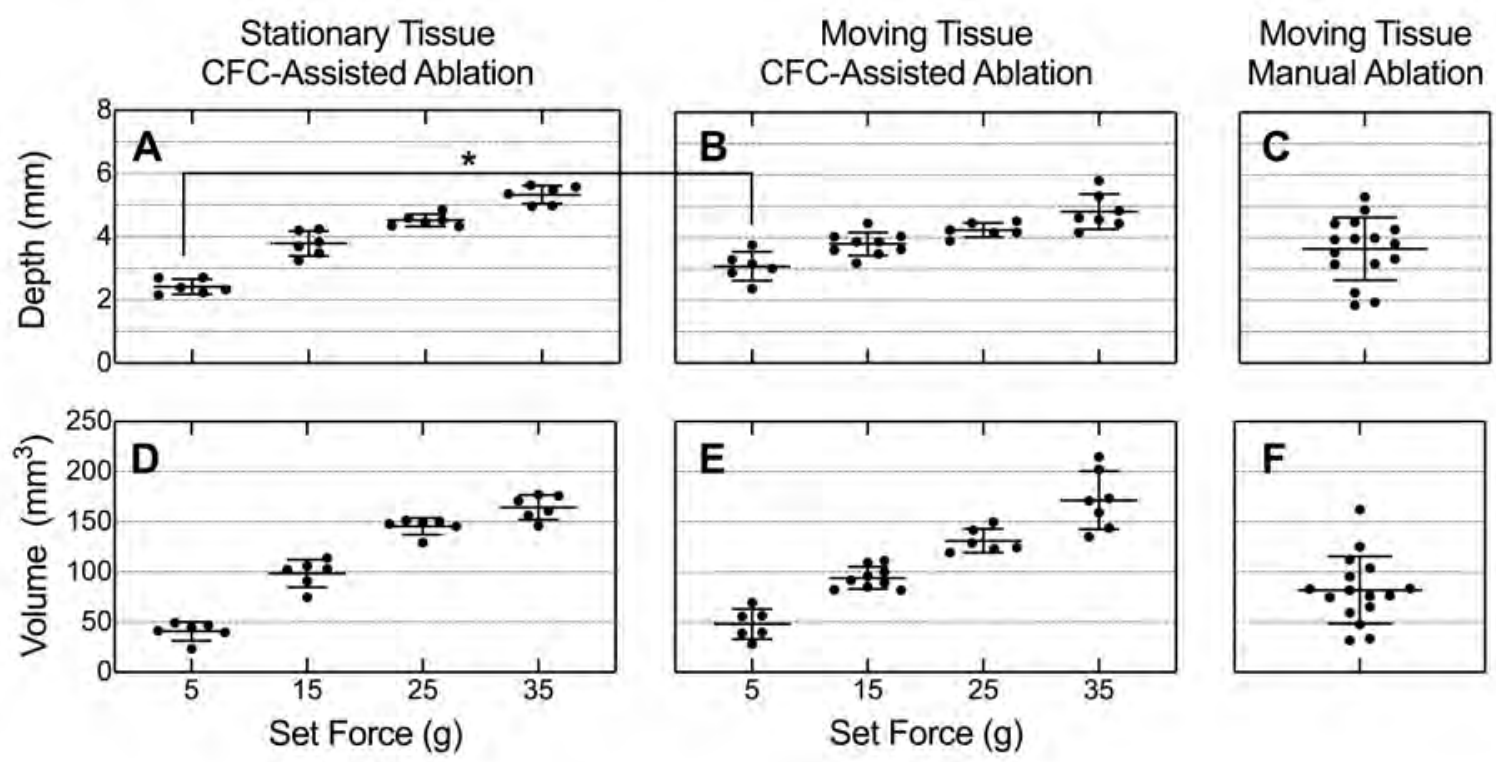

Figure 8. Measured lesion depth and volume are presented for CFC-assisted lesion creation on stationary tissue (A and D) and on moving tissue (B and E). For comparison depth and volume are also presented in panels $(\mathrm{C})$ and $(\mathrm{F})$ for lesions created under manual intervention (i.e. no control); these lesions were generated while the phantom was moving with the same motion profile as the controlled lesions generated in (B and E). For the same set force, no statistical differences between lesions volumes delivered to moving tissue and lesions delivered to stationary tissue were observed. Note that retrospectively analysed average force values for the manually delivered ablations $(\mathrm{C}$ and $\mathrm{F})$ demonstrated a similar relationship between depth/volume and achieved contact force, as expected; however, unlike the CFC-assisted case, the force level could not be set prospectively by the operator. 